\title{
Title
}

\section{MicroRNA function transitions from regulating developmental genes to transposable elements during the maturation of pollen}

\author{
Authors \\ Cecilia Oliver ${ }^{1}$, Maria Luz Annacondia ${ }^{1}$, Zhenxing Wang ${ }^{1}$, R Keith Slotkin ${ }^{2,3}$, \\ Claudia Köhler ${ }^{1}$ and German Martinez ${ }^{1}$ \\ Affiliations \\ 1. Department of Plant Biology, Swedish University of Agricultural Sciences and \\ Linnean Center of Plant Biology, Uppsala, Sweden \\ 2. Donald Danforth Plant Science Center, St. Louis, MO, United States of \\ America \\ 3. Division of Biological Sciences, University of Missouri Columbia
}

\begin{abstract}
microRNAs play important roles to control the development of eukaryotic organisms. Both animal and plant microRNAs are essential for the spatiotemporal regulation of development but together with this role, plant microRNAs also control transposable elements and stimulate the production of epigenetically-active small interfering RNAs. This last role is evident in the plant male gamete containing structure, the male gametophyte or pollen grain, but how the dual role of plant microRNAs is integrated during its development is unknown. Here, we provide a detailed analysis of microRNA dynamics during pollen development and their genic and transposable element targets using small RNA and mRNA cleavage (PARE) high-throughput sequencing. Furthermore we uncover the microRNAs loaded in the two main Argonaute proteins in the mature pollen grain, AGO1 and AGO5. Our results indicate that the developmental progression from microspore to mature pollen grain is characterized by a reprogramming from microRNAs focused on the control of development to microRNAs regulating transposable element control.
\end{abstract}

\section{Main}

Small non-coding RNAs control essential gene regulatory networks in eukaryotes at the transcriptional and postranscritional level. This broad term includes different classes of small RNAs (sRNAs) that have different biogenesis pathways, roles and cellular distribution but (in general) use sequence complementarity to recognize their target RNAs and silence their transcription and/or inhibit their translation ${ }^{1}$. The improvement of sequencing technologies has enabled to uncover the role of novel classes of sRNAs but also to understand better their cellular distribution and their roles during different stages of development of an organism or a tissue. According to their origin sRNAs can be classified in microRNAs (miRNAs), small interfering RNAs (siRNAs), piwiinteracting RNAs (piRNAs) or tRNA-derived sRNAs (tRFs), among others (for a recent review see: ${ }^{2}$ ). 
In the case of plants, the sRNome is monopolized by two classes of sRNAs: siRNAs and miRNAs ${ }^{3}$. These two types of sRNAs have different biogenesis pathways and functions. siRNAs are the result of the processing of an RNADEPENDENT RNA POLYMERASE (RDR)-produced double stranded RNA by Dicer-like proteins (DCL), mainly DCL4, DCL2 and DCL3. This leads to the production of double stranded sRNAs of between 21 and 24 nucleotides (nts) of which one of the strands will be selectively incorporated into an Argonaute (AGO) protein $^{4}$. On the other hand, miRNAs originate from MIRNA genes that produce non-coding transcripts with high self-complementarity that fold into a short hairpin structure. This hairpin is cleaved by DCL1 into a double-stranded sRNA of 21-22 nts in length. One of these sRNAs will then be selectively loaded into AGO1 and form the RISC complex, which uses the sRNA sequence to target mRNAs with perfect or imperfect sequence homology. In plants, this targeting normally leads to the cleavage of the mRNA, but can also induce translational repression ${ }^{4,5}$. Both miRNAs and SiRNA regulate a diversity of processes including development, defense, reproduction and genome stability. However, generally, siRNAs regulate heterochromatin and development/defense via DNA methylation and secondary sRNAs respectively, while miRNAs are associated with the regulation of development through the posttranscriptional control of transcription factor mRNAs ${ }^{4}$. Nevertheless these two sRNA classes are intertwined in certain aspects of development. For example, regulation of auxin signaling by the generation of trans-acting siRNAs is coordinated by miRNAs ${ }^{6}$. In plants, miRNAs also play a role in genome protection through the initial targeting of transposable elements (TEs) and generation of secondary siRNAs from their transcripts ${ }^{7,8}$. Interestingly, one of the miRNAs targeting TEs, miR845, is involved in the generation of TE-derived siRNAs in the male gametophyte and mediate genome dosage response ${ }^{9}$. This diversity of miRNA-regulated processes highlights their elasticity as regulatory molecules.

The functional versatility of miRNAs is especially important during reproduction, where cells face the duality of carrying out a very specific developmental program. In other organisms like zebrafish ${ }^{10}$, mouse $^{11}, C$. elegans ${ }^{12}$ or $D$. melanogaster ${ }^{13}$ miRNAs do not only have a differential accumulation pattern in sperm, but play important roles in sperm maturation, fertilization and postfertilization events. However, little is known about how miRNA activity might 83 shape the transcriptome during pollen development. Strong ago1 and dc/1 mutants have different reproductive abnormalities and reduced seed set ${ }^{14-16}$, which points to an important role of miRNAs during reproduction in plants.

89

Previous reports of Arabidopsis pollen sRNAs have focused on the analysis of the accumulation of these only in the mature pollen grain ${ }^{16,17}$. Here we analyze in depth the contribution of miRNAs to the sRNA population during the different

90 stages of pollen grain development, their loading into AGO proteins and their

91 target mRNAs. Overall, our work suggests that miRNAs involved in epigenetic

92 regulation, like miR845, are enriched at later stages of pollen grain development 
93 correlating with their preferential loading in AGO5. In contrast, miRNAs targeting mRNAs from genes involved in development decrease their accumulation during pollen development. This coincides with increased expression of their target genes at pollen maturity, which are mainly involved in pollen grain germination. Additionally, we identify a group of miRNA-regulated TEs in the pollen grain. In summary, this work shows that miRNAs modulate both the transcriptional and epigenetic reprogramming of the pollen grain.

\section{Results}

102

\section{Dynamic accumulation of miRNAs during pollen development}

105

106

107

To understand the potential changes in development during the transition leading to the mature pollen grain, we focused on analyzing miRNA accumulation at four different stages of pollen development (uninuclear, binuclear, trinuclear and mature pollen grain, representative pictures shown in Figure $1 \mathrm{a}$ and Supplementary Figure 1). Using density centrifugation we isolated four different developmental stages of pollen as previously described ${ }^{18}$. We isolated total RNA, prepared and sequenced sRNA libraries from two biological replicates for each of these stages (Supplementary Figure 1 and Supplementary Table 1). The total miRNA accumulation profiles between the different developmental stages did not reveal striking differences between the stages (Figure 1b); we only found a slight increase in $22 \mathrm{nt}$ miRNAs in trinuclear and mature pollen grain in comparison to uni- and binuclear (Figure 1b). The analysis of qualitative differences in the miRNA populations between our samples (Figure 1c) revealed that the majority of miRNA families are present in all our libraries (233 miRNAs); however, we also detected specific miRNAs in each developmental stage, including 23 miRNAs in the mature pollen grain and 13, 7, and 5 in the uni-, biand trinuclear stages, respectively (Supplementary Table 2). We further analyzed the quantitative changes experienced by the most highly accumulating miRNAs (Figure 1d). The comparison between the accumulation level of the top seven accumulating miRNA families (representing more than $90 \%$ of all sRNAs in uni-, bi- and tricellular pollen and $77 \%$ of the mature sRNAs) revealed that there is a striking progressive decrease in the accumulation of some miRNA families during pollen development. In particular, miRNAs involved in developmental processes, like miR156, miR161.1, miR159, miR166, and miR162 (Figure 1d) decrease at later stages of pollen development. In comparison, the relative levels of miR845 increase substantially during pollen development until occupying close to $45 \%$ of the all sRNAs at pollen maturity (Figure 1d). We confirmed this decrease in the accumulation of miRNAs for miR156 and miR161.1 by Northern blot (Figure 1e). 
bioRxiv preprint doi: https://doi.org/10.1101/864017; this version posted December 4, 2019. The copyright holder for this preprint (which was not certified by peer review) is the author/funder, who has granted bioRxiv a license to display the preprint in perpetuity. It is made available under aCC-BY-NC-ND 4.0 International license.

a.
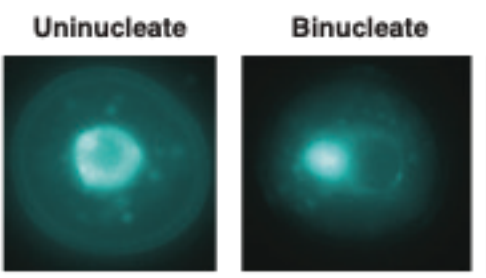

Trinucleate

Mature

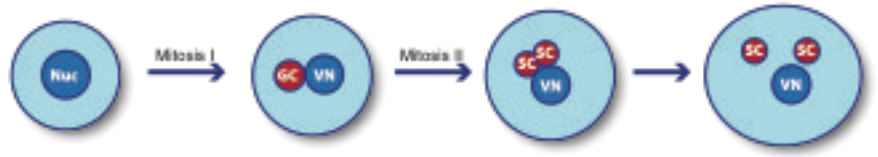

b.

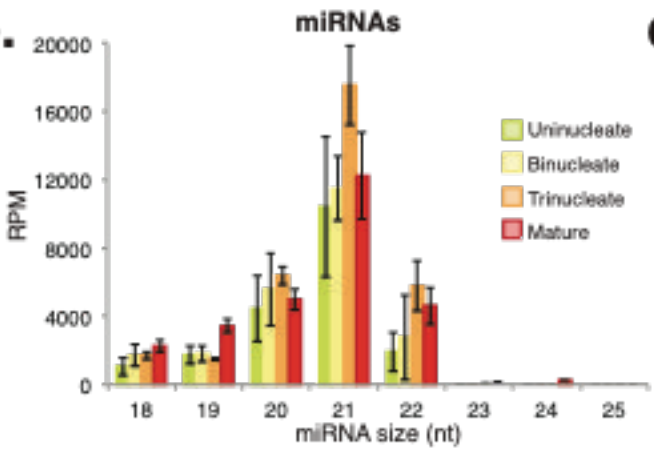

C.

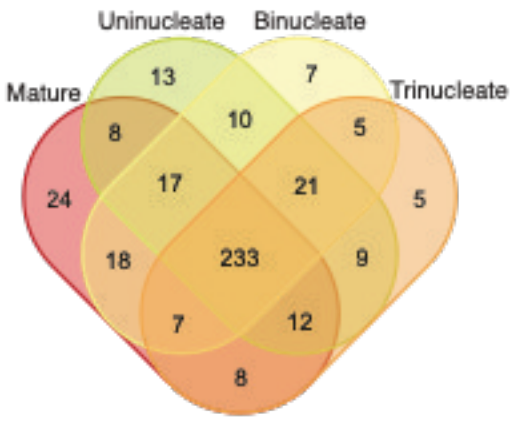

d.
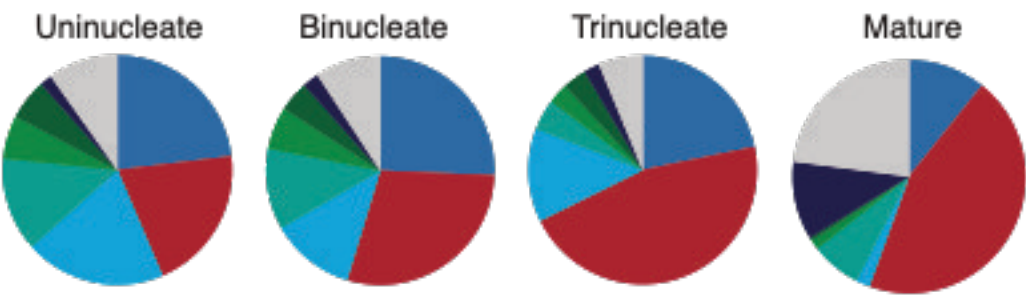

miA845

DiR156

miA161. 1

miA159

amin166

mir162

miA158

Oners

e.
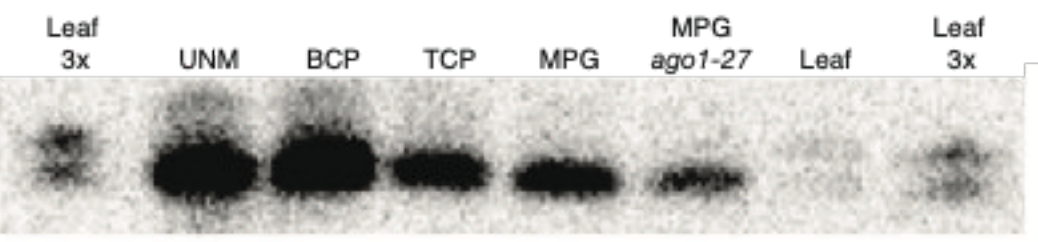

miR156

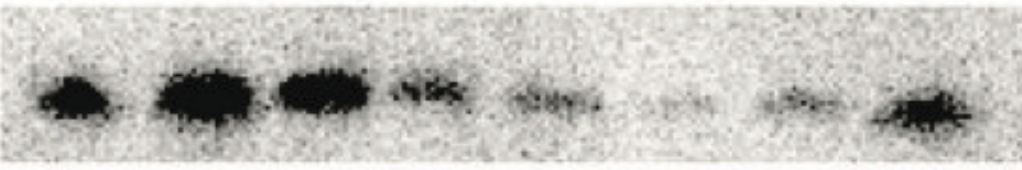

miR161.1

Figure 1. miRNome dynamics during pollen development: a) Representative images of the pollen developmental stages analyzed here. b) microRNA size distribution and accumulation for the developmental stages shown in a. c) Venn diagram showing the common and developmental stage-specific miRNAs for the stages indicated. d) Pie charts depicting the accumulation of main miRNAs during pollen development for each developmental stage. e) Northern blot showing the decrease in accumulation of two developmentally related miRNAs miR156 and miR161.1. The U6 small nuclear RNA was used as a control for RNA loading. 


\section{AGO1 and AGO5 loading explains miRNA enrichment in the mature pollen grain}

miRNA loading into AGO proteins determines their effect at the cellular level ${ }^{19}$. Several AGO proteins have been reported to be active in the male gametophyte, including AGO1, AGO2, AGO4, AGO5, and AGO9 16,20-22, but the sRNA populations loaded into them have not been studied. To shed light into the characteristics of the RISC complexes in the pollen grain, we analyzed the sRNAs bound to the main miRNA-related AGO proteins expressed in the pollen grain: AGO1 and AGO5 (Supplementary Figure 2). In the mature pollen grain, these two AGOs have a different cellular localization; while AGO1 is located in the vegetative nucleus and in the vegetative cell (VC) $)^{23}$, AGO5 accumulates in the sperm cell (SC) cytoplasm ${ }^{16}$. We investigated the cellular localization of both AGOs during pollen grain development and found that AGO1 is present already in the cytoplasm of unicellular pollen at the uninuclear stage and this expression pattern is maintained in the VC until the mature stage (Figure 2a). On the other hand, AGO5 was only detectable in the GC/SCs at the late binuclear/early trinuclear stage (Figure 2a). Next, we identified the sRNAs loaded into both AGOs by sequencing of sRNAs that were immunoprecipitated using AGOspecific antibodies. In line with their predicted role, we detected enrichment for miRNAs in the immunoprecipitated sRNAs compared to their input controls (Figure 2b). Both AGOs shared a proportion of their respective miRNomes (54.5\% and $61.3 \%$ for AGO1 and AGO5, respectively, Figure 2c), in particular both had a strong preference to load miR845 family members $(37.3 \%$ and $71.3 \%$ of the total miRNome for AGO1 and AGO5 respectively). AGO1 also loaded a substantial fraction of miRNAs with well-known roles in developmental processes, like miR158 (12.4\%), miR159 (9.5\%), miR156 (8.5\%), miR403 (6\%), and miR168 (4.8\%), while AGO5 loaded only a small fraction of developmentalrelated miRNAs, like miR156 (5.9\%) or miR158 (5.6\%) (Figure 2d). This different miRNA-loading pattern might reflect the different roles of both AGOs in relation to their cellular localization, with AGO1 being required to regulate the developmental program and post-transcriptional activity of TEs in the VC ${ }^{20,24}$, AGO5 might mediate specifically TE control in the SCs ${ }^{9}$. This correlates with the known role of the $\mathrm{VC}$ in the regulation of pollen development and germination ${ }^{25}$. In summary, both, the cellular localization of AGO1 and AGO5 and their loaded miRNAs correlate with two different programs mediating the regulation of development and TEs respectively. 
bioRxiv preprint doi: https://doi.org/10.1101/864017; this version posted December 4, 2019. The copyright holder for this preprint (which was not certified by peer review) is the author/funder, who has granted bioRxiv a license to display the preprint in perpetuity. It is made available under aCC-BY-NC-ND 4.0 International license.
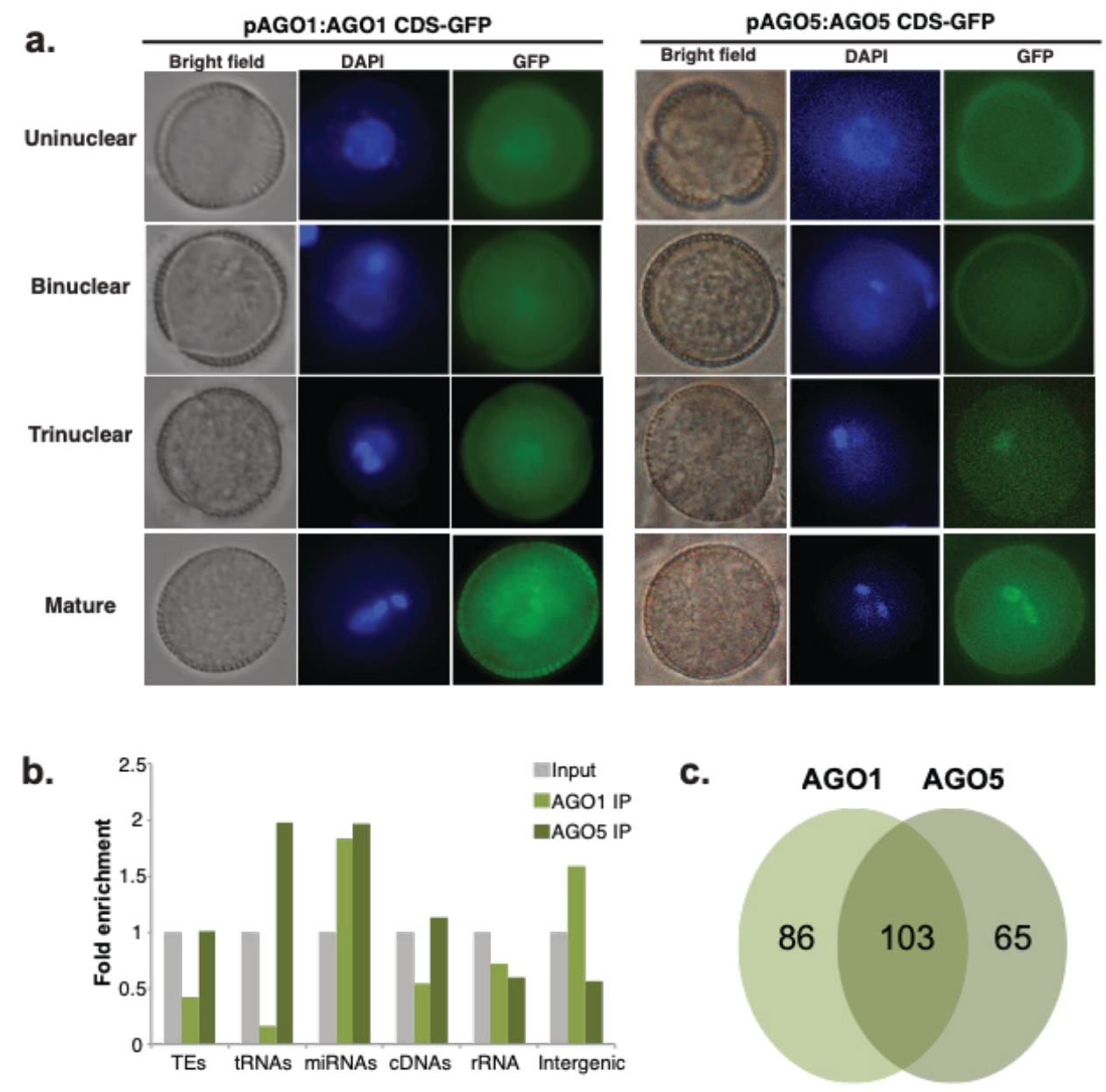

\section{d. AG01 IP}

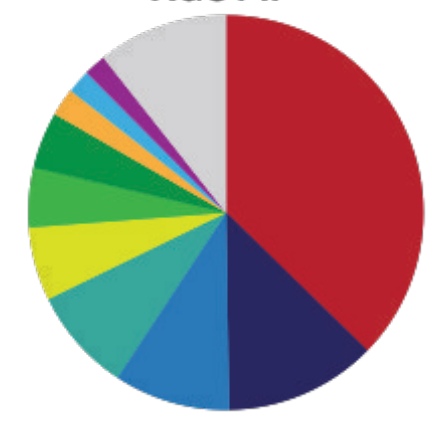

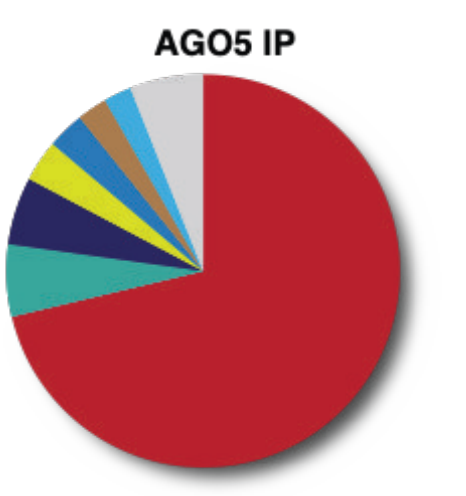

Figure 2: Comparison of AGO1 and AGO5 loaded miRNAs. a) Analysis of the cellular localization of AGO1 and AGO5-GFP fusion proteins during pollen development. b) Analysis of the enriched categories for sRNAs between 18 and 28 nts for AGO1 and AGO5 immunoprecipitated sRNAs compared to their respective input control. c) Venn diagram showing the number of common and exclusive miRNAs for each AGO protein. d) Pie chart depicting the miRNAs loaded preferentially on each of the AGO proteins under study. 
203

204

205

206

207

208

209

210

211

212

213

214

215

216

217

218

219

220

221

222

223

224

225

226

227

228

229

230

231

232

233

234

235

236

237

238

239

\section{Inhibition of miRNA activity affects pollen development}

To evaluate the level of influence of miRNAs on pollen grain development, we aimed to inhibit their activity at late stages of pollen development where AGO1 and AGO5 primarily accumulate (Figure 2a). Strong AGO1 mutant alleles fail to develop viable gametes ${ }^{14}$. We hypothesize that overexpression of a viral silencing suppressor using specific promoters would drive a cell-specific reduction in AGO1 activity as previously reported ${ }^{20}$. The Tombusvirus silencing suppressor P19 is a well-studied protein that inhibits miRNA/miRNA* duplex action $^{26}$. Accordingly, we expressed $\mathrm{P} 19^{27}$ fused to RFP under the control of the KRP6 promoter to drive the expression at late stages of development of the pollen grain $\mathrm{VC}^{28}$ (Figure 3a). KRP6pro::P19-RFP transgenic lines had defects in pollen development with many of the mature pollen grains aborted at maturity (Figure $3 b$ ) and inhibition of pollen grain germination (Figure 3c). In brief, inhibition of miRNA activity in the male gametophyte VC supports a role of VC miRNAs in the posttranscriptional regulation of genes required for pollen development.

\section{AGO1 and AGO5 are required for the triploid block response}

Gametic sRNAs establish hybridization barriers in different species ${ }^{29,30}$. In plants, polyploidization establishes hybridization barriers due to unbalanced expression of imprinted genes in the endosperm ${ }^{31-35}$, in a phenomenon known as the triploid block ${ }^{36}$. In Arabidopsis, the triploid block is established upon crosses of a pollen donor forming $2 \mathrm{n}$ pollen with a diploid maternal plant. Depletion of the major Pol IV subunit NRPD1A or the miRNA gene MIR845B suppresses the triploid block response ${ }^{9,37-39}$. To test whether the miRNA populations loaded in AGO1 or AGO5 are responsible for establishing the triploid block, we created tetraploid versions of the AGO1 weak allele ago1-27 and of the AGO5 alleles ago5-1 and ago5-5 and performed crosses with a wt diploid mother. The results of those pollinations revealed that paternal tetraploid ago127 weakly, but nevertheless significantly increased triploid seed viability (Figure $3 \mathrm{~d}$ and e). Similarly, paternal tetraploid ago5-1 and ago5-5 significantly increased triploid seed viability and seed germination (Figure $3 \mathrm{~d}$ and e) to a similar level as ago1, suggesting that both redundantly function in the triploid block response. Thus, paternal AGO1 and AGO5 are part of the triploid block response potentially through their loading of microRNA family members. 


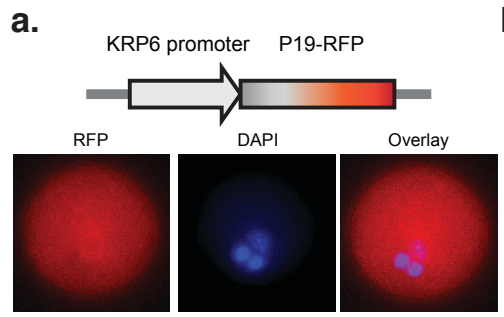

b.

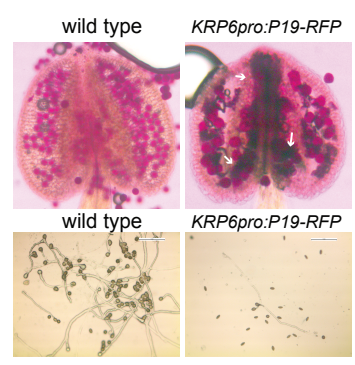

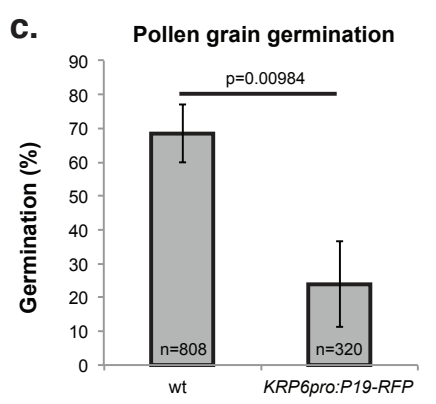

e.

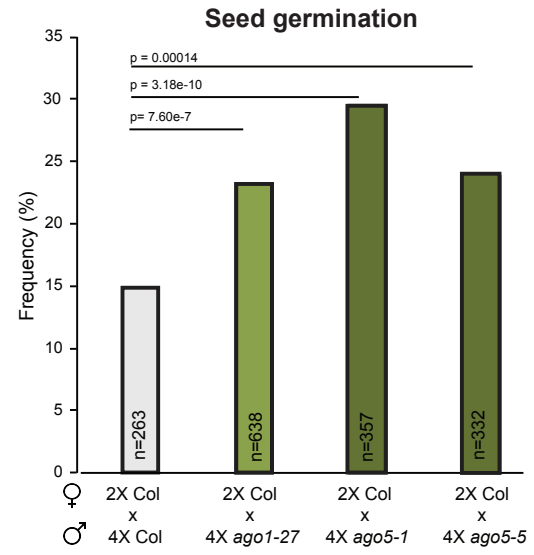

Seed germination d.

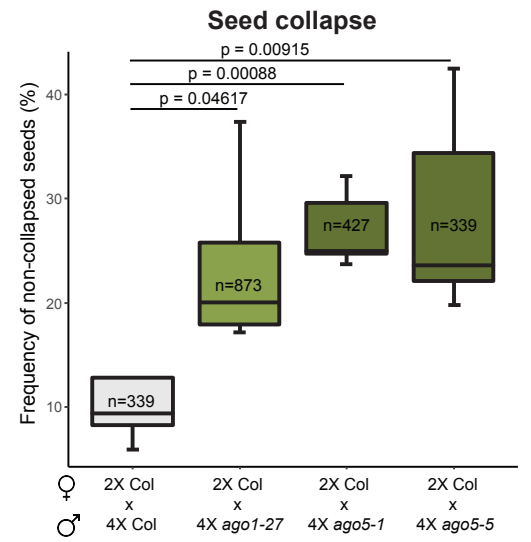

Figure 3: Inhibition of miRNA activity in the pollen grain leads to developmental defects of pollen grain development and inhibition of germination. a) Diagram of the construct used to express the viral silencing suppressor P19 in the mature pollen grain and representative image of its accumulation in mature pollen grains. b) Representative pictures of the analysis of pollen defects by Alexander staining and in vitro germination for wt and P19 transgenic plants. Aborted pollen grains clutches are indicated with white arrows. c) Pollen grain germination percentages for wt and KRP6pro:P19-GFP transgenic line. Number of individual pollen grain measurements $(n)$ is shown inside of each bar. Error bars represent the standard deviation values for the three bioreplicates analyzed. $P$ value is the result of a standard t-test with 2 tails and unequal variance. d) Frequency of non-collapsed and e) germinated seeds derived from crosses of wt (2xCol) maternal parents with $4 x$ mutants of indicated genotypes. T-test and Chi-squared test were performed in $\mathrm{E}$ and $F$, respectively. Number of individual seed measurements $(n)$ is shown inside of each bar. Whiskers in the box plots extent to the maximum and minimum values.

\section{Genome-wide analysis of miRNA-regulated transcripts in the pollen grain}

To uncover miRNA-regulated transcripts in the pollen grain, we generated and sequenced PARE libraries from mature pollen grains from Col-0 wild type plants and from mature rosette leaves, as previously described ${ }^{40}$ (Supplementary Table 1). PARE is a technique that targets cleaved mRNAs with a polyA tail but without a 5' cap for library preparation and sequencing. A comparison of miRNA-targeted mRNAs between leaf and pollen highlighted the tissue specificity of miRNA regulation, as only $21.5 \%$ of pollen miRNA-targeted genes were also miRNAregulated in the leaf (Fig 4a). These target mRNAs are regulated by miRNAs that are both shared with leaf (68\% of all pollen miRNAs) and pollen-specific (representing $32 \%$ of the total active miRNAs). A global analysis of pollen miRNA-regulated transcripts revealed that the majority are associated with developmental processes related to transport, cell organization and biogenesis, 
signal transduction, and response to stress (Supplementary Figure 4 and

270 Supplementary Table 3).

271

272

Pollen miRNA target mRNAs are involved both in pollen grain development and pollen tube germination and include well-known regulators of these processes such as SK32, AtbZIP34 and AFB3 involved in pollen development ${ }^{41-43}$, or MYB97, MYB101, and SYP131 involved in pollen tube germination ${ }^{44,45}$. miRNAs with a higher number of targeted transcripts included juvenile-to-adult phase transition related $\mathrm{miR} 172^{46}$ and $\mathrm{miR} 159^{47}$ (13.5 and $10 \%$ of targeted genes) and also the pollen specific miR5021 (10\% of targeted genes) (Figure $4 \mathrm{~b})$. miRNA targets included classic miRNA-regulated genes, such as the TAS genes or miR172-targeted transcription factors APETALA2 (AP2) and TARGET OF EARLY ACTIVATION TAGGED (EAT) 2 (TOE2) (Supplementary Figure 4). We also detected pollen-specific targeting events like the targeting of SKU5 SIMILAR 12 (SKS12) by miR5021 or ETHYLENE-INSENSITIVE3 (EIN3) by miR5015 (Supplementary Figure 3 and Supplementary Table 4). In other organisms, miRNA targeting in the gametes increases the stability of targeted transcripts ${ }^{48}$. We explored if a similar scenario may apply to Arabidopsis. The distribution of 5'$P$ end reads in a $100 \mathrm{nt}$ window from the predicted target site shows that most of these targeting events resulted in cleavage of the target RNAs without evidence of ribosomal stalling (Figure 4c). All these evidences show that miRNA activity in the pollen grain induces the cleavage of transcripts involved in pollen grain development and germination.

To test this observation, we obtained homozygous mutants for two of the genes specifically regulated by miRNAs in pollen identified in our PARE analysis: SKS12 (AT1G55570, mutant termed sks12-1, Supplementary Figure 4) and EIN3 (AT3G20770, ein3-1) ${ }^{49}$ (Figure 4d) and evaluated the ability of their mature pollen grain to germinate in vitro (Figure 4e). Measurement of pollen tube length and germination rate after $16 \mathrm{hrs}$ of incubation indicated that while only ein3-1 was affected in the rate of pollen germination (Figure $4 \mathrm{~g}$ ), both mutants were impaired in pollen tube growth (Figure 4f). Thus, we conclude that miRNAs

300 regulate developmental processes that are important for pollen development and 301 pollen grain germination. 
bioRxiv preprint doi: https://doi.org/10.1101/864017; this version posted December 4, 2019. The copyright holder for this preprint (which was not certified by peer review) is the author/funder, who has granted bioRxiv a license to display the preprint in perpetuity. It is made available under aCC-BY-NC-ND 4.0 International license.

a.

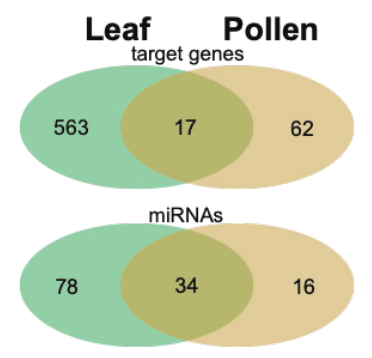

b.

d.
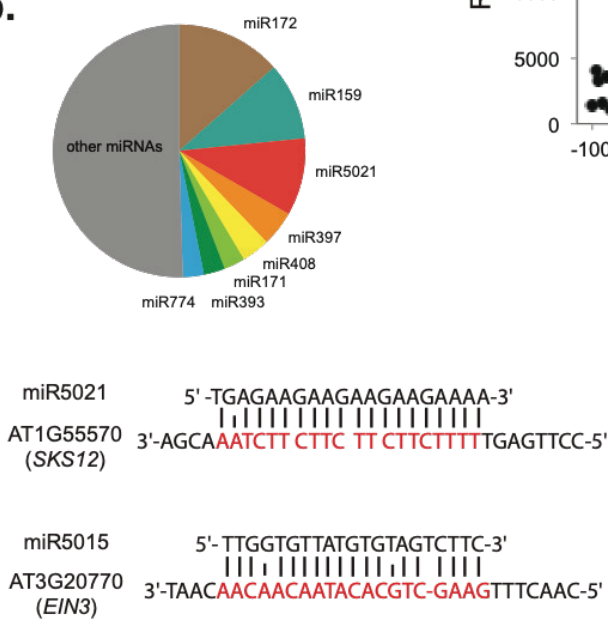

f.

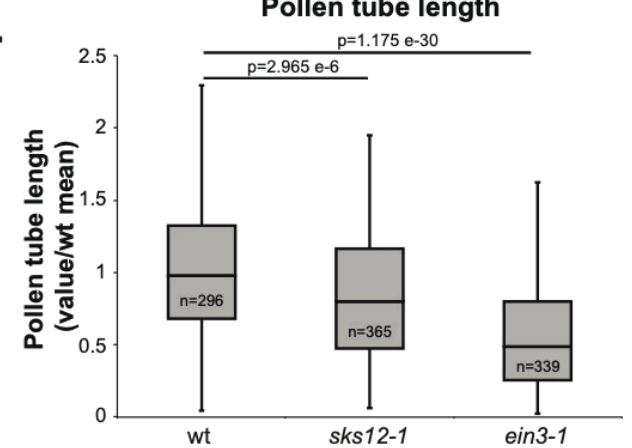

c.

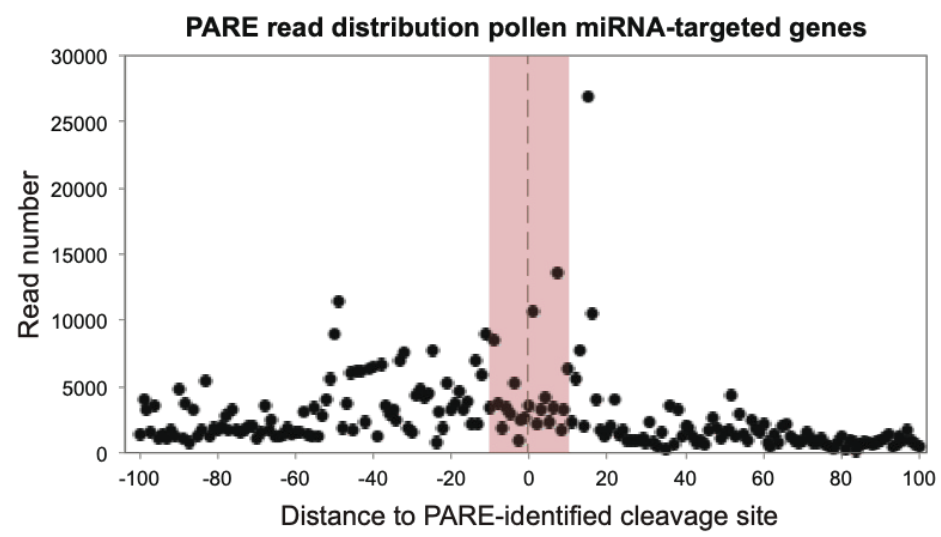

e.

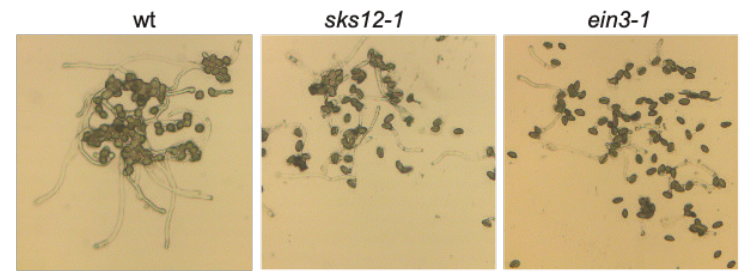

g.

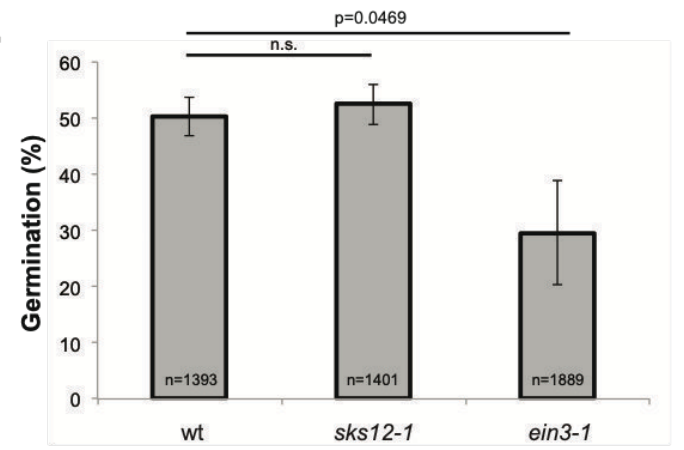

Figure 4: Analysis of miRNA genic targets in the mature pollen grain identified by PARE sequencing: a) Venn diagrams showing the common and tissue specific target genes and active miRNAs for the tissues analyzed. b) Pie chart showing highly represented miRNA target sites on PARE confirmed miRNA target genes. c) Distribution of 5' ends of PARE reads around the predicted cleavage site (located at coordinate 0 in the $X$ axis) in a $100 \mathrm{nt}$ window. Red zone represents the physical position covered by the bound miRNA. d) Examples of two miRNA targets in our PARE analysis: SKS12-miR5021 and EIN3-miR5015. e) Representative pictures of pollen grain germination for wt and the sks12-1 and ein3-1 mutants. f) Length of the pollen tube and g) percentage of germination for in vitro germinated pollen grains for the genotypes indicated. Number of individual pollen grain measurements $(n)$ is shown inside of each bar. Error bars in $h$ represent the standard deviation values for the three bioreplicates analyzed. $\mathrm{P}$ value is the result of a standard t-test with 2 tails and unequal variance. Whiskers in the box plots extent to the maximum and minimum values. 
319 miR845-targeted TEs progressively decrease their level of 24 nt sRNAs during pollen development

miRNAs have been identified as important posttranscriptional regulators of $\mathrm{TEs}^{7,9}$. In particular, the miR845 family is involved in the biogenesis of epigenetically-active siRNAs (easiRNAs) through the targeting of the primer binding site (PBS) of TEs $^{9}$. This miRNA family is composed by two members, miR845a and miR845b, which are 21 and 22 nts in length, respectively 9 . Analysis of their presence in our pollen development sRNA libraries showed that, during pollen development, the two members of the miR845 family increased their accumulation, especially miR845a which increased by 2.7 fold (Figure 5a). Both miRNAs did not seem to be affected by fluctuations of the processing precision and were of the expected size at all stages of development (Supplementary Figure 5). Interestingly, the 5' terminal nucleotide of miR845a and $b$ ( $C$ and $T$ respectively, Figure $5 b)$ suggests a preferential loading in AGO5 and $A G 01$, respectively ${ }^{50}$. We analyzed if this predicted differential loading was detectable in our AGO1 and AGO5 IP sRNA libraries and, indeed, AGO5 showed a clear preferential loading of miR845a (62\% of AGO5 IPed sRNA sequences, Figure 5c).

Members of the miR845 family were prosed to trigger Pol IV-dependent easiRNA biogenesis during meiosis or early gametogenesis ${ }^{9}$. Analysis of easiRNAs derived from miR845-targeted TEs indicates that easiRNAs accumulate to high levels already at the unicellular stage (Figure 5d). As expected from its preferential loading in AGO1, miR845b targeted-TEs produce easiRNAs earlier and to a greater extent than miR845a targets (Supplementary Figure 5). Interestingly, during pollen grain development there is a gradual transition from a majority of $24 \mathrm{nts}$ at the unicellular stage to a majority of 22 nts at pollen maturity (Figure 5d-e). This shows that, most probably, miR845-dependent easiRNA biogenesis takes place after meiosis progressively during the two rounds of pollen mitosis. This tendency of losing $24 \mathrm{nt}$ sRNAs during pollen grain development and gaining 21/22 nt sRNAs is common for all TEs (Figure 5f-g), revealing that similar mechanisms to miR845 targeting might exist for all TEs. Unfortunately, TE-targeted by miR845 could not be confirmed using our PARE sequencing (Supplementary Table 4) since PARE libraries are prepared from polyadenylated transcripts ${ }^{40}$ and miR845 targets non-polyadenylated Pol IV transcripts ${ }^{9}$. This fact is in agreement with the prediction that miR845 family members target exclusively Pol IV transcripts ${ }^{9}$. Overall, these results show that TEs tend to loss 24 nts while gaining 21/22 nt sRNAs during pollen development in correlation with an increase in the accumulation of miR845 family members.

359

360

361 
a.

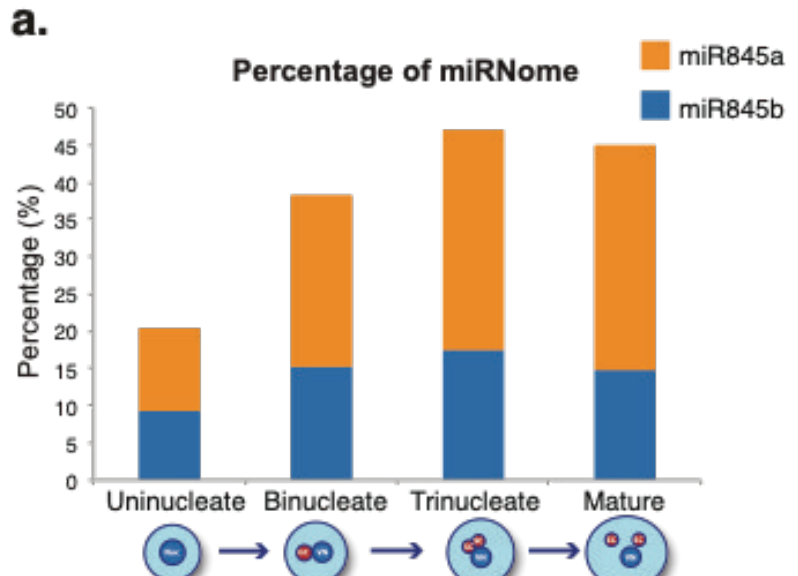

b. miR845a

5'-cggcucugauaccaauugaug

miR845b

5'- UCGCUCUGAUACCAAAUUGAUG

c.

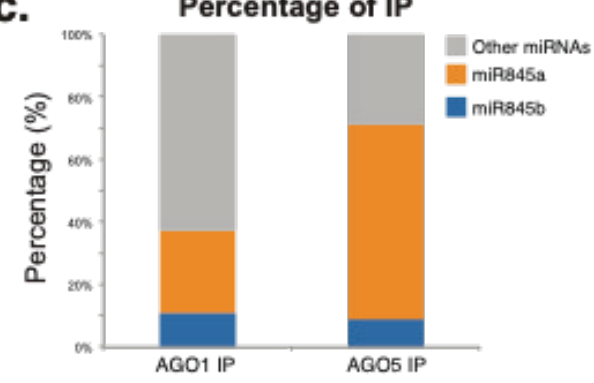

d.

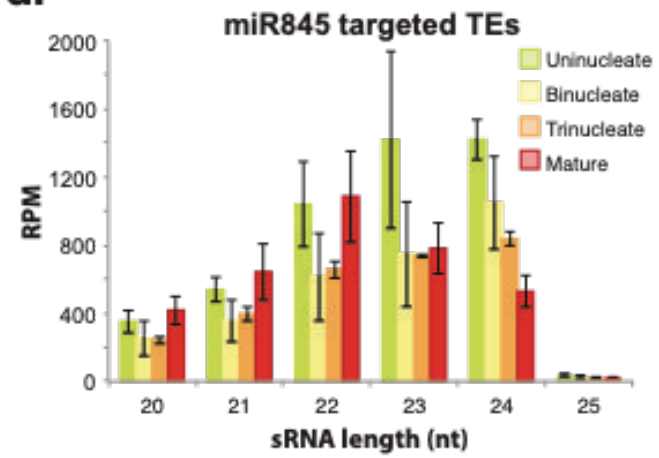

f.

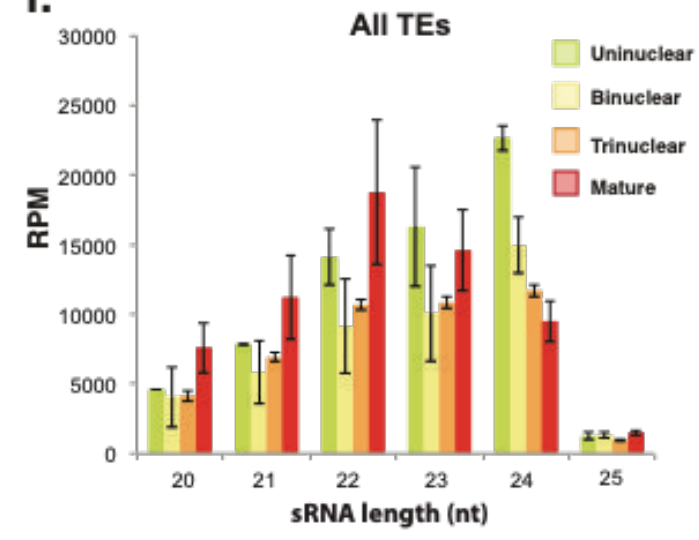

e.

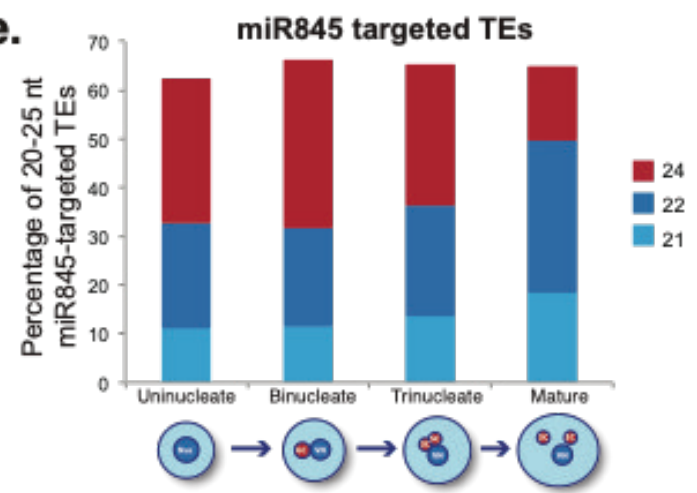

g.

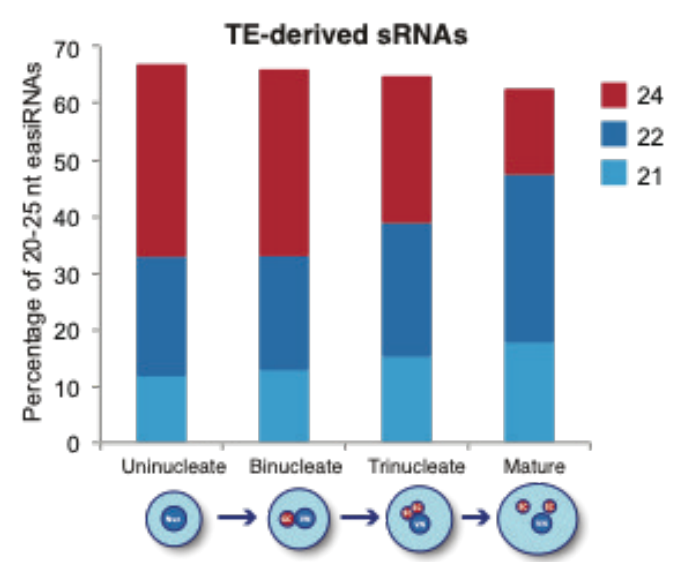

Figure 5: Analysis of miR845 dynamics and its target TEs during pollen development. a) Accumulation percentages for miR845a and $b$ during pollen development. b) Sequence comparison of miR845a and b with the 5'nucleotide highlighted. c) Percentage of the impact of miR845 a and b on total AGO1 and AGO5 immunoprecipitated miRNAs. d) Accumulation size profile of TE-derived siRNAs of predicted miR845targeted TEs. e) Accumulation of 21,22 and 24 nt sRNAs from miR845-targeted TEs during pollen development shown as percentages of total 20-25 nt sRNAs derived from those TEs. f) Accumulation profile of all TE-derived sRNAs. g) Accumulation of 21,22 and $24 \mathrm{nt}$ sRNAs from TEs during pollen development shown as percentages of total $20-25$ nt easiRNAs.

374 
375 PARE sequencing analysis identified a pool of Pol Il-transcribed and 376 miRNA-regulated TEs

377

378

379

380

381

382

383

384

385

386

387

388

389

390

391

392

393

394

395

396

397

398

399

400

401

402

403

404

405

406

407

408

409

410

411

412

413

414

415

416

417

418

419

420
Although our PARE libraries could not be used to identify miR845-targeting events, they allowed the identification of miRNA-targeted polyadenylated TE transcripts. We identified a number of miRNA-targeted TEs (Supplementary Table 4), strongly suggesting that Pol II transcribes these TEs. Interestingly, these TEs were targeted mostly by pollen-specific miRNAs like miR5021, miR5658 and miR5645 (that together represent 58\% of the targeting events identified) (Figure 6a). Similar to the targeting of genic mRNAs, distribution of reads in a 100 nt window from the PARE-identified cleavage site for TEs revealed a clear preference for RNA cleavage (Figure 6b). Most of these TEs localized to euchromatic regions $(57.5 \%$, Figure $6 \mathrm{~d}$ ) and belonged to the MuDR, Copia, En-Spm and Gypsy families (82\% of total miRNA-targeted TEs, Figure 6c). miRNA-targeted TEs dramatically lost 24 nts during the transition from unicellular to bicellular pollen (Figure 6e), revealing that their regulation is different from the rest of TEs (Figure 5f).

Next, we analyzed the levels of DNA methylation of PARE-identified miRNAtargeted TEs during pollen development ${ }^{51}$ and its potential connection to their sRNA levels using publicly available data (Supplementary Table 5). miRNAtargeted TEs have significantly lower levels of $\mathrm{CHH}$ methylation at the unicellular stage compared to the rest of TEs (Figure 6f), pointing to their dependence on the RdDM pathway to retain sRNA-based $\mathrm{CHH}$ methylation. At the mature developmental stage, these TEs retain significant higher levels of CG methylation in the VN compare to other TEs (Figure $6 \mathrm{~g}$ ) while maintain low levels of $\mathrm{CHH}$ methylation in the SCs (Supplementary Figure 6). Altogether this may indicate that this group of TEs is not a target of DME-mediated demethylation in the VN. Subsequently, analysis of DNA methylation in the VN of dme mutants confirmed that miRNA-targeted TEs are indeed not targeted by DNA glycosylases (Figure 6h), which translates into maintenance of low $\mathrm{CHH}$ methylation in the SCs (Supplementary Figure 6). Altogether, our data points to the existence of Pol IItranscribed TEs during pollen epigenetic reprogramming that are not targeted by DME but are regulated by miRNAs. 


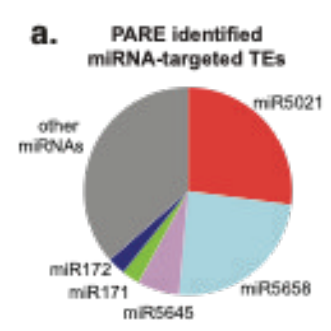

d.

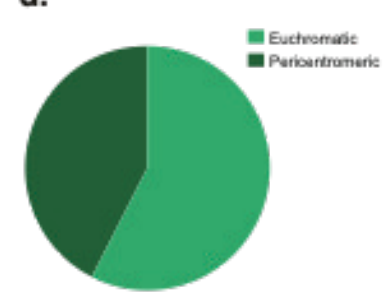

b.

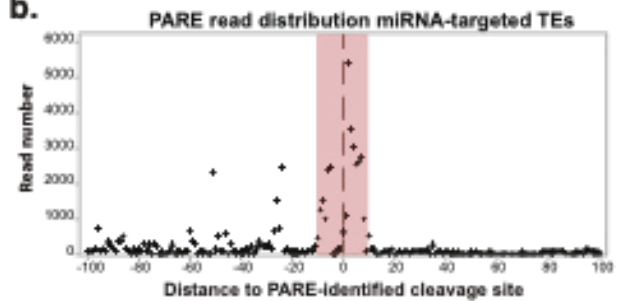

e.

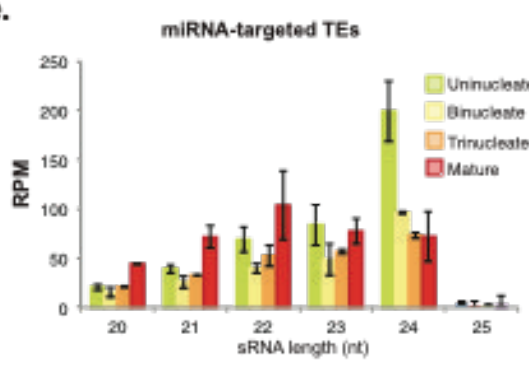

c.

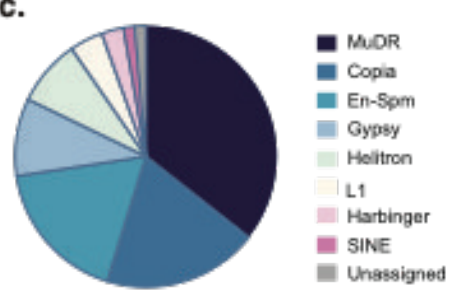

f.
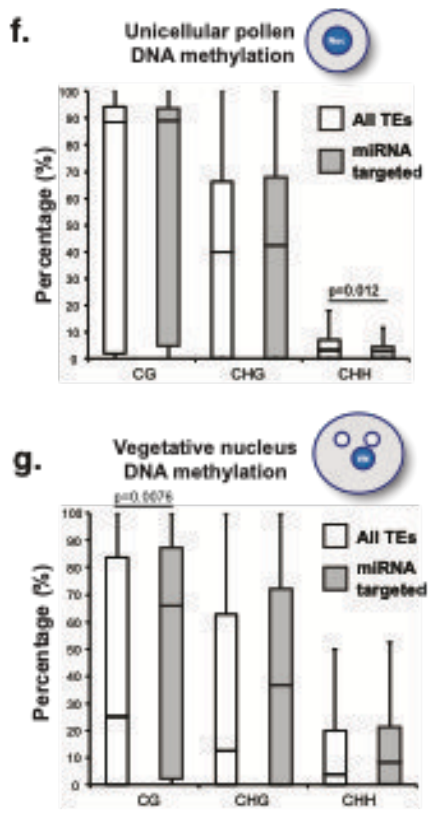

h.
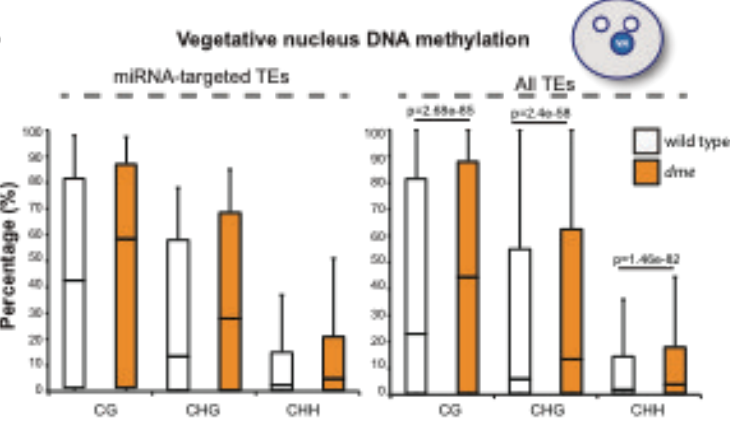

Figure 6: Analysis of miRNA TE targets in the mature pollen grain identified by PARE sequencing. a) Pie chart showing highly represented miRNA target sites on PARE confirmed miRNA-targeted TEs. b) Global distribution of 5' ends of PARE reads around the predicted cleavage site for TEs (located at coordinate 0 in the $X$ axis) in a $100 \mathrm{nt}$ window. Red zone represents the physical position covered by the mRNA-bound miRNA. c) Family categorization and d) genomic distribution of miRNA-targeted TEs. e) sRNA accumulation size profile for TEs predicted to be targeted by miRNAs and transcribed by Pol II. f-g) Levels of cytosine methylation for the different contexts (CG, $\mathrm{CHG}$ and $\mathrm{CHH}$ ) in the unicellular pollen grain (f) and vegetative nucleus (g) for all TEs (white boxes) or miRNA-targeted TEs (grey boxes). h) Levels of cytosine methylation for the different contexts (CG, CHG and $\mathrm{CHH}$ ) in the vegetative nucleus for miRNA-targeted TEs (left panel) or all TEs (right panel) in wild type (white boxes) and dme (orange boxes). In all graphs, $\mathrm{P}$ value is the result of a standard t-test with 2 tails and unequal variance. Only significant differences between measurements are highlighted in the graphs. Whiskers in the box plots extent to the maximum and minimum values.

\section{The miRNome adjusts during pollen development to adapt the regulation of genic and TE targets}

Finally, to understand the role of the identified active miRNAs during pollen development, we studied in detail their accumulation patterns in our sRNA libraries from different stages of pollen development. During pollen maturation, the majority of miRNAs regulating genes and TEs (and the common miRNAs between both groups, Supplementary Figure 7a) maintained or decreased their level of accumulation $(65.7 \%$, Figure $7 a$ and Supplementary Figure $7 b-c)$. This decrease was especially evident at the tricellular stage where the SCs appear in the VC and AGO5 accumulation was evident (Figure 2A). Interestingly, the members of the miR845 family followed the opposite trend, with a progressive accumulation during pollen development (Figure 7a). In parallel with the 
447 decrease of Pol II-active miRNA accumulation, their genic targets increased their 448 expression towards maturity of the pollen grain (Figure 7c). The level of 449 expression of these miRNA-target genes was even maintained during pollen grain germination (Figure $7 \mathrm{c}$ ), indicating that miRNAs regulating genic products in the pollen grain have an important role in the overall regulation of transcripts available for pollen development and germination.

The analysis of the two populations of active miRNAs present in our AGO immunoprecipitated libraries helped to understand to a better extent their role during pollen development (Figure 7b). AGO1 tended to load a mix of miRNAs involved both in the regulation of development (36.6\%) and TEs (44\%) (Figure $7 b)$. On the other hand AGO5 loads a majority of miRNAs involved exclusively in the regulation of TEs (82\%, Figure $7 \mathrm{~b})$. In summary, overall, our analysis shows that during pollen development there is a transition from a diverse miRNA pool that regulates both development and Pol II-transcribed TEs, probably loaded in AGO1, to a miRNA pool that, at maturity, controls Pol IV-transcribed TEs monopolized by miR845 and with preferential loading in AGO5.
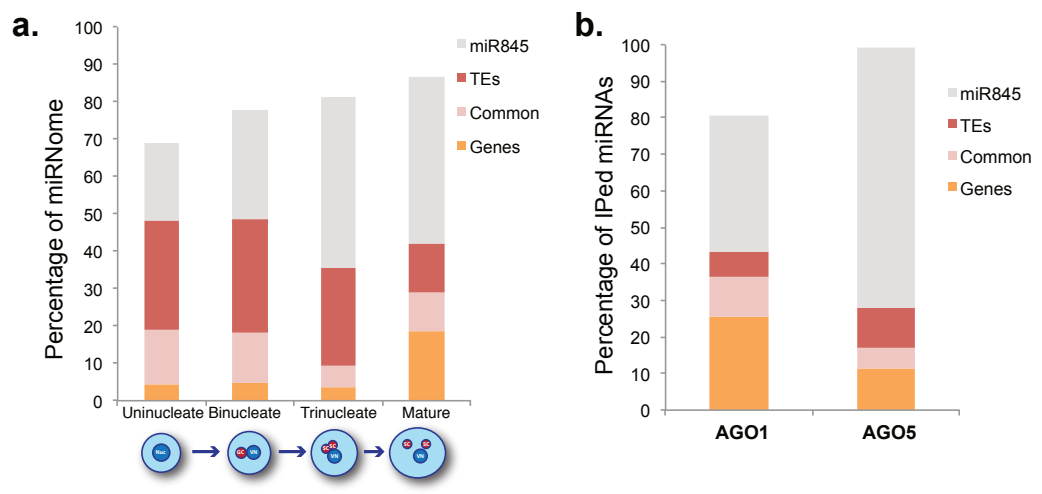

c.

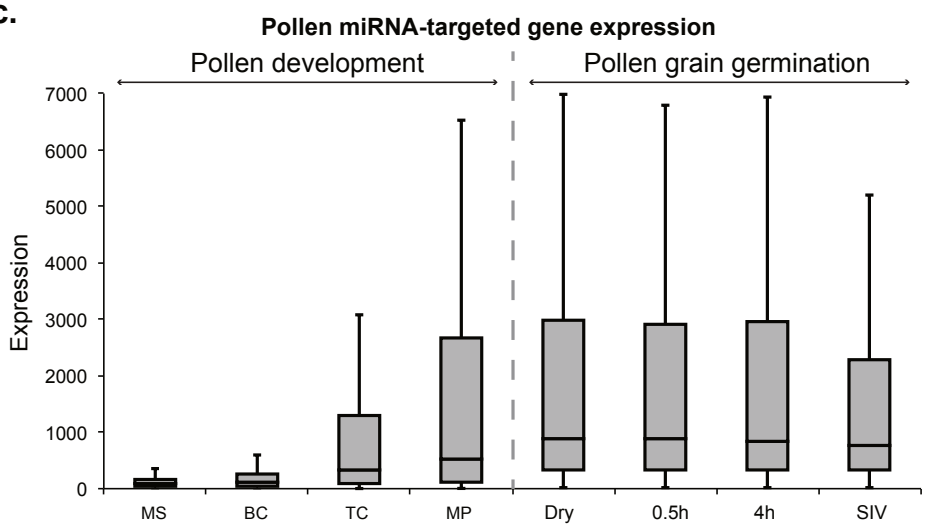

Figure 7. a) Distribution of miR845 family and PARE-identified miRNAs targeting TEs, miRNAs or both (termed common) during pollen development. Accumulation values are represented as percentage of the total miRNome. b) Presence of miR845 family and PARE-identified miRNAs targeting TEs, miRNAs or both (termed common) in AGO1 and 5 immunoprecipitated sRNAs. c) Level of expression of miRNA target genes during pollen development and pollen grain germination present in the ATH1 microarray (MS= microspore, $\mathrm{BC}=$ bicellular pollen, $\mathrm{TC}=$ tricellular pollen, $\mathrm{MP}=$ mature pollen grain, $\mathrm{Dry}=$ Dessicated mature pollen, $0.5 \mathrm{~h}=$ In vitro-germinated pollen grains after 30 minutes, $4 \mathrm{~h}=$ In vitro-germinated pollen grains after 4 hours and $\mathrm{SIV}=$ Pollen tubes grown through the stigma and style). Whiskers in the box plots extent to the maximum and minimum values. 


\section{Discussion}

Through the use of pollen stage separation combined with high-throughput sRNA sequencing, PARE sequencing and the characterization of several characteristics of pollen grain development, we have (1) identified the characteristics of the miRNome during pollen grain development, (2) determined the miRNA populations loaded into the main AGO proteins in the pollen grain, AGO1 and AGO5, (3) identified miRNA targets (both TEs and genes) and (4) identified the involvement of both AGO1 and AGO5 in the triploid block. Our data reveal that the miRNome experiences a reprogramming during pollen development, transitioning from a miRNome mainly involved in developmental control to a miRNA population focused on the transcriptional control of TEs.

The pollen grain undergoes both a transcriptional and epigenetic reprogramming during its transition to maturity, but whether the first is a consequence of the latter is unknown ${ }^{24,51,52}$. Our data indicates that miRNAs also experience a reprogramming, which could influence the transcriptional and epigenetic changes taking place in this tissue. Reduction of miRNAs in the pollen grain via the expression of the P19 viral silencing suppressor strongly reduced pollen grain viability and germination (Figure 3 ). Indeed our identification of miRNA target mRNAs in the pollen grain of Arabidopsis (Figure 4 and 6) through PARE sequencing shows their importance in the regulation of both genes and TEs.

In this tissue, genes targeted by miRNAs had a higher level of expression in the mature pollen stage and were involved in processes related with pollen germination (Figure 7c). Although this might suggests that gametic miRNAs increase the stability of transcripts, similar as previously observed in C.elegans ${ }^{48}$, our PARE data indicates that most probably the miRNA targeting events identified here induce the cleavage of their target mRNAs (Figure 4c), showing that developmental miRNA targets in pollen are expressed at high levels and miRNA targeting dampens their expression (Figure 7a-c, model shown in Supplementary Figure 8). As a proof of concept, we analyzed the defects in pollen grain germination experienced by two miRNA target genes identified in our analysis: SKS12 and EIN3 (Figure 4d-g).

Additionally we have explored the influence of the miRNome on the epigenetic regulation of TEs in the pollen grain. During pollen grain development the miR845 family members (miR845a and b) increase in abundance (Figure 1d). This increase likely results in the preferential loading of these two miRNAs in AGO5, a highly abundant AGO protein in the sperm cells (Figure 2a). miR845 members have been proposed to target Pol IV transcripts of several retrotransposons and induce the production of 21/22 easiRNAs from those transcripts ${ }^{9,37}$. Our analysis indicates that indeed simultaneous to the increase in the accumulation of miR845 members during pollen development, there is a 518 parallel decrease of $24 \mathrm{nt} \mathrm{sRNAs}$ from their targets and a progressive increase of $51921 / 22 \mathrm{nt}$ sRNAs (Figure $5 \mathrm{~d}-\mathrm{e}$ ), potentially a consequence of their targeting. 
520 Nevertheless, this needs to be tested since our PARE sequencing excluded non-

521 polyadenylated RNAs. Interestingly, the preferential loading of miR845 by AGO5

522 correlates with low levels of $\mathrm{CHH}$ methylation in the $\mathrm{SCs}^{51}$. Due to the proposed

523 role of miR845 in targeting of Pol IV transcripts ${ }^{9}$, we speculate that increased

524 presence and activity of this miRNA in the SCs upon AGO5 loading impairs $\mathrm{CHH}$

525 methylation establishment. Interestingly, both AGO1 and AGO5 are able to

526 weakly rescue the triploid block-induced seed collapse (Figure 3d-e), which might

527 be the consequence of their redundant ability to load miR845 family members.

528

529

530

531

532

533

534

535

536

537

538

539

540

541

542

543

544

545

546

547

548

549

550

551

552

553

554

555

556

557

558

559

560

561

562

563

Together with this, our PARE sequencing and analysis has identified a series of transposons that were transcribed by Pol II and regulated by miRNAs (Figure 6). These transposons are likely regulated primarily by the RdDM pathway, due to their strong loss of $24 \mathrm{nt}$ sRNAs and low values of $\mathrm{CHH}$ methylation in the unicellular stage (Figure 6c-f). Furthermore, miRNA-targeted TEs in the pollen grain seemed to not DME-mediated demethylation in the VN since they keep significantly higher CG methylation levels compared to the rest of TEs in the VN and their C-methylation values in the $\mathrm{VN}$ are not affected in a DME mutant (Figure 6f-h). We speculate that miRNA-targeting for these TEs might be a safeguard mechanism to avoid their spurious expression.

In summary, our work highlights the relevance of miRNAs for the developmental and epigenetic events that occur during the pollen grain development. The pollen grain needs to face the duality of reprogramming the transcriptome and epigenome of the newly established gametes in the sperm cells, while accomplishing a complex developmental program that culminates in the germination of the pollen tube and the successful transfer of the male gametes to the female gametophyte. Like in plants, in mouse and human cell lines changes in DNA methylation and miRNAs are an important part of the reprogramming of cells to pluripotency ${ }^{53-55}$, which might be also linked to a potential miRNA control of DNA methylation, cell cycle transitions and regulation of apoptosis ${ }^{56,57}$. Our work highlights that the complexity of the orchestration of the miRNome is not exclusive of mammalian reprograming for pluripotency, but also takes place during reproductive reprogramming in plants.

\section{Methods:}

\section{Plant material}

Plants were grown under standard long day conditions at $22^{\circ} \mathrm{C}$. The mutant alleles used in this study were ein3-1 (NASC accession number: N8052), sks121 (SALK_061973) and ago1-27. The KRP6pro:P19-RFP transgene construction, plant transformation and selection were performed as described in Martinez et al (2016). Primers used for cloning are shown in Supplementary Table 6.

564

Total RNA, sRNA Northern blot AGO immunoprecipitation and sRNA/PARE library construction 
565 Total RNA was isolated using TRIzol reagent (Life Technologies). For microRNA 566 Northern blot detection, $5 \mu \mathrm{g}$ of total RNA were loaded in each lane for pollen 567 developmental stage Northern blots. sRNA gel electrophoresis, blotting, and 568 cross-linking were performed as described in Pall et al. $(2008)^{58}$. The AGO1 and 569 AGO5 proteins were immunoprecipitated using commercially available polyclonal AGO1 and AGO5 antibodies (Agrisera AB). AGO immunoprecipitated sRNA libraries were constructed as indicated in McCue et al (2012) adapted to pollen tissue. PARE libraries were constructed following the protocol described in Zhai et al (2014) adapted to pollen tissue. All sRNA libraries were made using the NEBNext Small RNA Library Prep Set for Illumina (New England Biolabs) following the manufacturer instructions and using gel-enriched sRNAs as described in Martinez et al (2016). 581 fractions termed B1 (Unicellular), B3 (Bicellular), A3 (Tricellular). Pollen

582

583

584

585

\section{Pollen grain separation, germination, viability test and microscopy.}

Pollen grain separation was performed as described in Dupl'akova et al (2016). The pollen developmental stages used for sRNA sequencing correspond to the al $(2013)^{59}$. Each germination assay was performed in triplicates. Standard Alexander staining method was used to visualize pollen grain abortion as described in Alexander MP $(1969)^{60}$. Visualization of pollen grain germination and Alexander stained pollen grains was performed in a Leica DM RX microscope. For pollen grain fluorescence, pollen grains of T3 plants were mounted on slides containing $50 \%$ glycerol and analyzed under a Zeiss Axioplan or a Leica DMI 4000 microscope fluorescence microscopes.

\section{Bioinformatic analysis}

sRNA libraries were trimmed using Trim Galore. Reads were aligned using bowtie with the command "bowtie $-f-t-v 2$ " that allows two mismatches. The TAIR10 version of the Arabidopsis genome and the miRbase version 21 were used in this analysis. Reads were normalized to reads per million to the total reads mapped to the Arabidopsis chromosomes. For PARE library analysis, miRNA cleavage events were identified using PARESnip ${ }^{61}$. For genome-wide plots of PARE reads, PARE libraries were aligned using bowtie to retain only perfectly matched reads (0 mismatches). The pericentromeric region limits was determined using the description from Copenhaver et al., $(1999)^{62}$.

\section{References}

1 Stefani, G. \& Slack, F. J. Small non-coding RNAs in animal development. Nat Rev Mol Cell Biol 9, 219-230 (2008).

2 Martens-Uzunova, E. S., Olvedy, M. \& Jenster, G. Beyond microRNA-novel RNAs derived from small non-coding RNA and their implication in cancer. Cancer Lett 340, 201-211 (2013).

610

3 Borges, F. \& Martienssen, R. A. The expanding world of small RNAs in plants. Nat Rev Mol Cell Biol 16, 727-741 (2015). 
6114 Axtell, M. J. Classification and comparison of small RNAs from plants.

$612 \quad$ Annu Rev Plant Biol 64, 137-159 (2013).

6135 Ma, X., Cao, X., Mo, B. \& Chen, X. Trip to ER: MicroRNA-mediated

614 translational repression in plants. RNA Biol 10, 1586-1592 (2013).

6156 Hunter, C. et al. Trans-acting siRNA-mediated repression of ETTIN and

616

617 ARF4 regulates heteroblasty in Arabidopsis. Development 133, 2973-2981

6187 Creasey, K. M. et al. miRNAs trigger widespread epigenetically activated

619

620 siRNAs from transposons in Arabidopsis. Nature 508, 411-415 (2014).

8 Surbanovski, N., Brilli, M., Moser, M. \& Si-Ammour, A. A highly specific

621

622

623 microRNA-mediated mechanism silences LTR retrotransposons of strawberry. Plant J 85, 70-82 (2016).

624

625

626

627

628

629

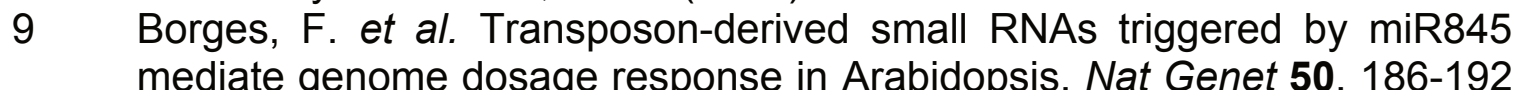
(2018).

10 Presslauer, C., Tilahun Bizuayehu, T., Kopp, M., Fernandes, J. M. \& Babiak, I. Dynamics of miRNA transcriptome during gonadal development of zebrafish. Sci Rep 7, 43850 (2017).

630

631

632

633

634

635

636

637

638

639

11 Yuan, S. et al. Sperm-borne miRNAs and endo-siRNAs are important for fertilization and preimplantation embryonic development. Development 143, 635-647 (2016).

12 McJunkin, K. \& Ambros, V. The embryonic mir-35 family of microRNAs promotes multiple aspects of fecundity in Caenorhabditis elegans. G3 (Bethesda) 4, 1747-1754 (2014).

13 Eun, S. H. et al. MicroRNAs downregulate Bag of marbles to ensure proper terminal differentiation in the Drosophila male germline. Development 140, 23-30 (2013).

640

14 Kidner, C. A. \& Martienssen, R. A. The role of ARGONAUTE1 (AGO1) in meristem formation and identity. Dev Biol 280, 504-517 (2005).

15 Jacobsen, S. E., Running, M. P. \& Meyerowitz, E. M. Disruption of an

641

642

643 RNA helicase/RNAse III gene in Arabidopsis causes unregulated cell division in floral meristems. Development 126, 5231-5243 (1999).

644

645

646

647

648

Borges, F., Pereira, P. A., Slotkin, R. K., Martienssen, R. A. \& Becker, J. D. MicroRNA activity in the Arabidopsis male germline. $J$ Exp Bot 62, 1611-1620 (2011).

17 Grant-Downton, R. et al. MicroRNA and tasiRNA diversity in mature pollen of Arabidopsis thaliana. BMC Genomics 10, 643 (2009).

649

650

18 Dupl'akova, N., Dobrev, P. I., Renak, D. \& Honys, D. Rapid separation of Arabidopsis male gametophyte developmental stages using a Percoll gradient. Nat Protoc 11, 1817-1832 (2016).

652 Hutvagner, G. \& Simard, M. J. Argonaute proteins: key players in RNA silencing. Nat Rev Mol Cell Biol 9, 22-32 (2008).

654 Martinez, G., Panda, K., Kohler, C. \& Slotkin, R. K. Silencing in sperm cells is directed by RNA movement from the surrounding nurse cell. Nat 655 Plants 2, 16030 (2016). 
65621 Oliver, C., Santos, J. L. \& Pradillo, M. Accurate Chromosome Segregation

657

658

659

660

661

662

663

664

665

666

667

668

669

670

671

672

673

674

675

676

677

678

679

680

681

682

683

684

685

686

687

688

689

690

691

692

693

694

695

696

697

698

699

at First Meiotic Division Requires AGO4, a Protein Involved in RNADependent DNA Methylation in Arabidopsis thaliana. Genetics 204, 543553 (2016).

22 Olmedo-Monfil, V. et al. Control of female gamete formation by a small RNA pathway in Arabidopsis. Nature 464, 628-632 (2010).

23 McCue, A. D., Nuthikattu, S., Reeder, S. H. \& Slotkin, R. K. Gene expression and stress response mediated by the epigenetic regulation of a transposable element small RNA. PLoS Genet 8, e1002474 (2012).

24 Slotkin, R. K. et al. Epigenetic reprogramming and small RNA silencing of transposable elements in pollen. Cell 136, 461-472 (2009).

25 Glockle, B. et al. Pollen differentiation as well as pollen tube guidance and discharge are independent of the presence of gametes. Development 145 (2018).

26 Chapman, E. J., Prokhnevsky, A. I., Gopinath, K., Dolja, V. V. \& Carrington, J. C. Viral RNA silencing suppressors inhibit the microRNA pathway at an intermediate step. Genes Dev 18, 1179-1186 (2004).

27 Csorba, T., Kontra, L. \& Burgyan, J. viral silencing suppressors: Tools forged to fine-tune host-pathogen coexistence. Virology 479-480, 85-103 (2015).

28 Kim, H. J. et al. Control of plant germline proliferation by SCF(FBL17) degradation of cell cycle inhibitors. Nature 455, 1134-1137 (2008).

29 Martienssen, R. A. Heterochromatin, small RNA and post-fertilization dysgenesis in allopolyploid and interploid hybrids of Arabidopsis. New Phytol 186, 46-53 (2010).

30 Senti, K. A. \& Brennecke, J. The piRNA pathway: a fly's perspective on the guardian of the genome. Trends Genet 26, 499-509 (2010).

31 Kradolfer, D., Wolff, P., Jiang, H., Siretskiy, A. \& Kohler, C. An imprinted gene underlies postzygotic reproductive isolation in Arabidopsis thaliana. Dev Cell 26, 525-535 (2013).

32 Wolff, P., Jiang, H., Wang, G., Santos-Gonzalez, J. \& Kohler, C. Paternally expressed imprinted genes establish postzygotic hybridization barriers in Arabidopsis thaliana. Elife 4 (2015).

33 Jiang, $\mathrm{H}$. et al. Ectopic application of the repressive histone modification H3K9me2 establishes post-zygotic reproductive isolation in Arabidopsis thaliana. Genes Dev 31, 1272-1287 (2017).

34 Wang, G., Jiang, H., Del Toro de Leon, G., Martinez, G. \& Kohler, C. Sequestration of a Transposon-Derived siRNA by a Target Mimic Imprinted Gene Induces Postzygotic Reproductive Isolation in Arabidopsis. Dev Cell 46, 696-705 e694 (2018).

35 Huang, F. et al. Mutants in the imprinted PICKLE RELATED 2 gene suppress seed abortion of fertilization independent seed class mutants and paternal excess interploidy crosses in Arabidopsis. Plant $J$ 90, 383395 (2017). 
70036 Kohler, C., Mittelsten Scheid, O. \& Erilova, A. The impact of the triploid block on the origin and evolution of polyploid plants. Trends Genet 26, 142-148 (2010).

703

37 Martinez, G. et al. Paternal easiRNAs regulate parental genome dosage in Arabidopsis. Nat Genet 50, 193-198 (2018).

704

38 Erdmann, R. M., Satyaki, P. R. V., Klosinska, M. \& Gehring, M. A Small RNA Pathway Mediates Allelic Dosage in Endosperm. Cell Rep 21, 33643372 (2017).

709

39 Satyaki, P. R. V. \& Gehring, M. Paternally Acting Canonical RNA-Directed DNA Methylation Pathway Genes Sensitize Arabidopsis Endosperm to Paternal Genome Dosage. Plant Cell 31, 1563-1578 (2019).

711

40 Zhai, J., Arikit, S., Simon, S. A., Kingham, B. F. \& Meyers, B. C. Rapid

712

713

714

715

716

717

718

719

720

721

722

723 construction of parallel analysis of RNA end (PARE) libraries for Illumina sequencing. Methods 67, 84-90 (2014).

41 Dong, X., Nou, I. S., Yi, H. \& Hur, Y. Suppression of ASKbeta (AtSK32), a Clade III Arabidopsis GSK3, Leads to the Pollen Defect during Late Pollen Development. Mol Cells 38, 506-517 (2015).

42 Gibalova, A. et al. AtbZIP34 is required for Arabidopsis pollen wall patterning and the control of several metabolic pathways in developing pollen. Plant Molecular Biology 70, 581-601 (2009).

43 Cecchetti, V., Altamura, M. M., Falasca, G., Costantino, P. \& Cardarelli, M. Auxin regulates Arabidopsis anther dehiscence, pollen maturation, and filament elongation. Plant Cell 20, 1760-1774 (2008).

44 Liang, Y. et al. MYB97, MYB101 and MYB120 Function as Male Factors That Control Pollen Tube-Synergid Interaction in Arabidopsis thaliana Fertilization. Plos Genetics 9 (2013).

725

726

727

728

729

730

731

732

733

734

735

736

737

738

739

740

74150

45 Slane, D., Reichardt, I., El Kasmi, F., Bayer, M. \& Jurgens, G. Evolutionarily diverse SYP1 Qa-SNAREs jointly sustain pollen tube growth in Arabidopsis. Plant Journal 92, 375-385 (2017).

$46 \mathrm{Wu}, \mathrm{G}$. et al. The sequential action of miR156 and miR172 regulates developmental timing in Arabidopsis. Cell 138, 750-759 (2009).

47 Guo, C. et al. Repression of miR156 by miR159 Regulates the Timing of the Juvenile-to-Adult Transition in Arabidopsis. Plant Cell 29, 1293-1304 (2017).

48 Dallaire, A., Frederick, P. M. \& Simard, M. J. Somatic and Germline MicroRNAs Form Distinct Silencing Complexes to Regulate Their Target mRNAs Differently. Dev Cell 47, 239-247 e234 (2018).

49 Kieber, J. J., Rothenberg, M., Roman, G., Feldmann, K. A. \& Ecker, J. R. CTR1, a negative regulator of the ethylene response pathway in Arabidopsis, encodes a member of the raf family of protein kinases. Cell 72, 427-441 (1993).

742

743 Mi, S. et al. Sorting of small RNAs into Arabidopsis argonaute complexes is directed by the $5^{\prime}$ terminal nucleotide. Cell 133, 116-127 (2008).

744 epigenetic inheritance via small RNA. Cell 151, 194-205 (2012). 
74552 Honys, D. \& Twell, D. Transcriptome analysis of haploid male

746

747

748

749

750

751

752

753

754

755

756

757

758

759

760

761

762

763

764

765

766

767

768

769

770

771

772

773

774

775

776

777

778

779

780

781

782

783

784

785

786

787

788

789

790 gametophyte development in Arabidopsis. Genome Biol 5, R85 (2004).

53 Anokye-Danso, F. et al. Highly efficient miRNA-mediated reprogramming of mouse and human somatic cells to pluripotency. Cell Stem Cell 8, 376388 (2011).

54 Lin, S. L. et al. Regulation of somatic cell reprogramming through inducible mir-302 expression. Nucleic Acids Res 39, 1054-1065 (2011).

55 Miyoshi, N. et al. Reprogramming of mouse and human cells to pluripotency using mature microRNAs. Cell Stem Cell 8, 633-638 (2011).

56 Fabbri, M. et al. MicroRNA-29 family reverts aberrant methylation in lung cancer by targeting DNA methyltransferases $3 \mathrm{~A}$ and 3B. Proc Natl Acad Sci U S A 104, 15805-15810 (2007).

57 Leonardo, T. R., Schultheisz, H. L., Loring, J. F. \& Laurent, L. C. The functions of microRNAs in pluripotency and reprogramming. Nat Cell Biol 14, 1114-1121 (2012).

58 Pall, G. S. \& Hamilton, A. J. Improved northern blot method for enhanced detection of small RNA. Nat Protoc 3, 1077-1084 (2008).

59 Rodriguez-Enriquez, M. J., Mehdi, S., Dickinson, H. G. \& Grant-Downton, R. T. A novel method for efficient in vitro germination and tube growth of Arabidopsis thaliana pollen. New Phytol 197, 668-679 (2013).

60 Alexander, M. P. Differential staining of aborted and nonaborted pollen. Stain Technol 44, 117-122 (1969).

61 Stocks, M. B. et al. The UEA sRNA Workbench (version 4.4): a comprehensive suite of tools for analyzing miRNAs and sRNAs. Bioinformatics 34, 3382-3384 (2018).

62 Copenhaver, G. P. et al. Genetic definition and sequence analysis of Arabidopsis centromeres. Science 286, 2468-2474 (1999).

\section{Acknowledgements}

The authors thank Professor Feng Qu for the P19 clone and Professor Michael Lenhard for his gift of the cellulosic membranes used for pollen grain germination. Sequencing was performed by the SNP\&SEQ Technology Platform in Uppsala. The facility is part of the National Genomics Infrastructure (NGI) Sweden and Science for Life Laboratory. The SNP\&SEQ Platform is also supported by the Swedish Research Council and the Knut and Alice Wallenberg Foundation.

\section{Author information}

\section{Funding}

Research in the G.M. group is supported by SLU, the Carl Tryggers Foundation and the Swedish Research Council (VR 2016-05410).

\section{Data access:}


791 Data can be accessed at the GEO accession number

792 reviewer token:

793

794 Supplementary information:

B1 (Unicellular stage)

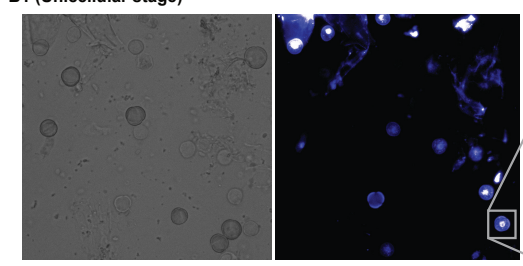

B3 (Bicellular stage)

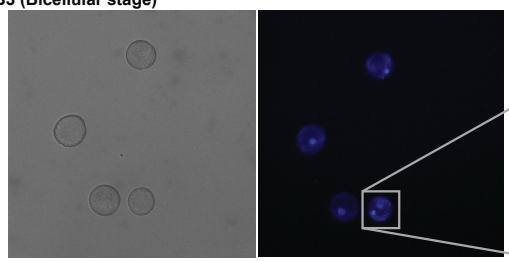

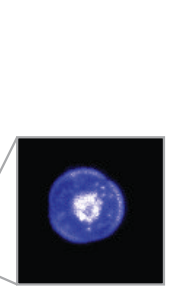

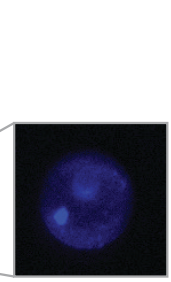

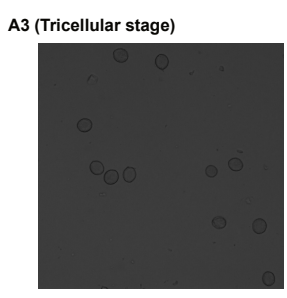

Mature Pollen Grain

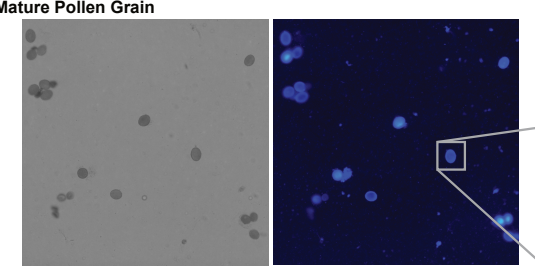

with the
795

796

797

798

799

800

801

802

803

804

Supplementary Figure 1. Representative pictures for each of the fractions of pollen developmental stages analyzed by sRNA high-throughput sequencing: B1 (Unicellular), B3 (Bicellular), A3 (Tricellular) and mature pollen grains.

a.

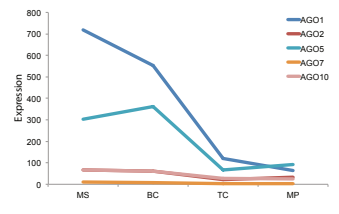

b.

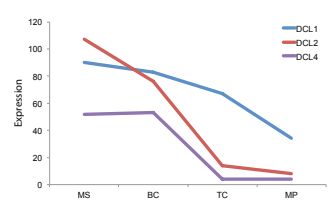

c.

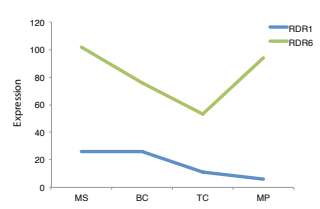

Supplementary Figure 2. Expression pattern of different RNA silencing components involved in miRNA-related pathways during pollen development: a) AGO, b) DCL and c) RDR genes. Data extracted from ATH1 microarray. MS= microspore, $\mathrm{BC}=$ bicellular pollen, $\mathrm{TC}=$ tricellular pollen and $\mathrm{MP}=$ mature pollen grain. 
a.

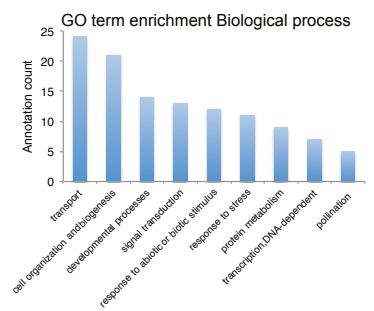

b.
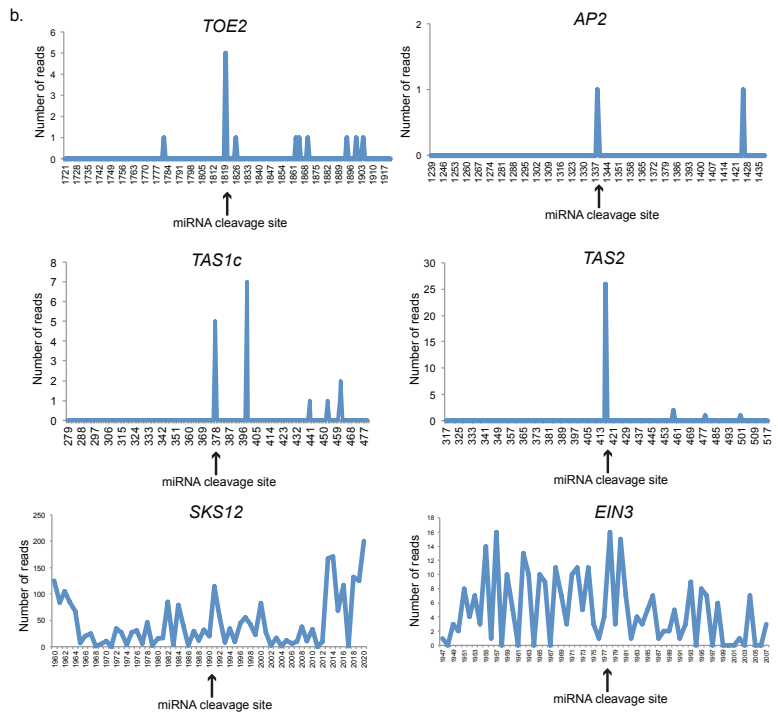

806

807

Supplementary Figure 3. a) Analysis of the enriched GO categories for pollen

808 specific miRNA-targeted genes. b) PARE read distribution along miRNA target

809 sites for representative miRNA-targeted genes in Arabidopsis: miR172-targeted genes TOE2 and AP2 and miR173-targeted TAS1c and TAS2 and the miRNAtargeted genes analyzed here: SKS12 and EIN3.

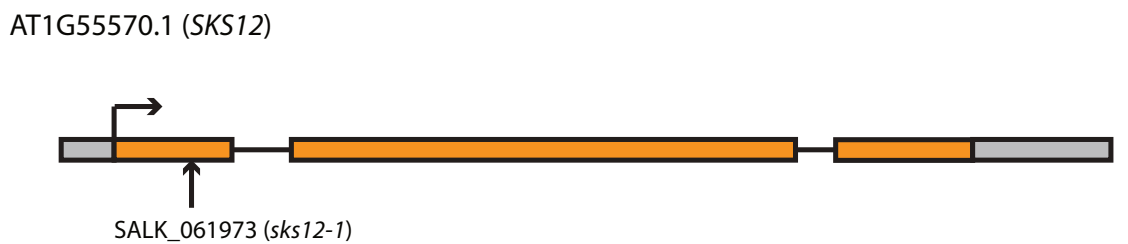

811

812 Supplementary Figure 4. Diagram showing the location of the T-DNA insertion 813 for the sks12-1 mutant analyzed in this study (SALK_061973). 
a.

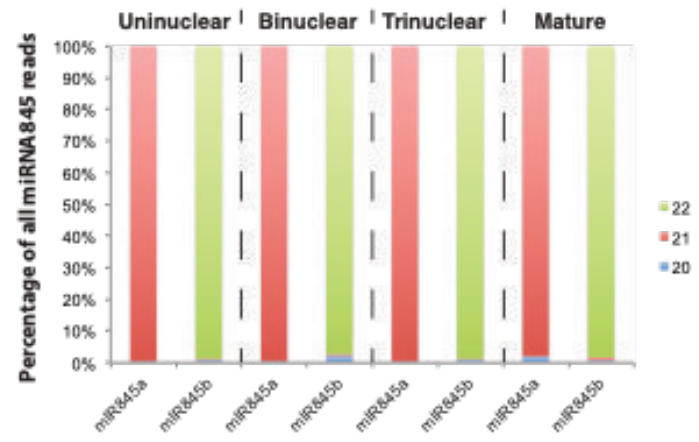

b.

814

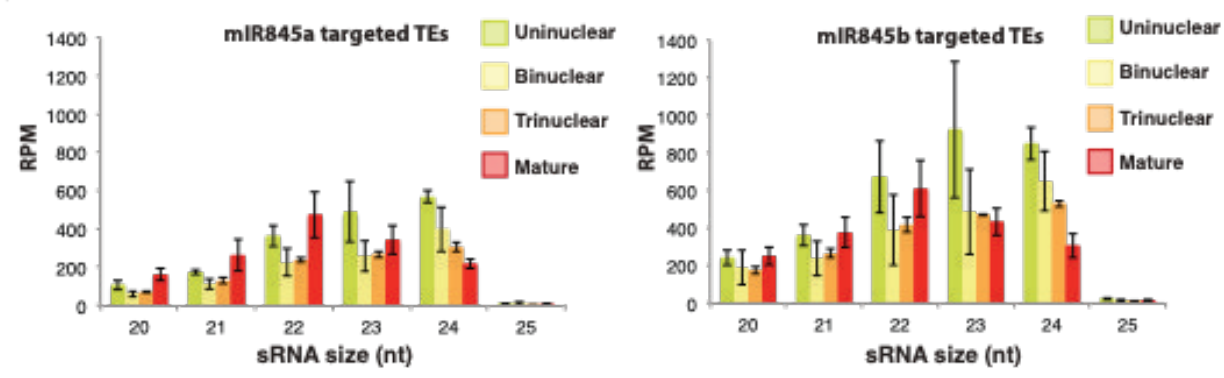

815 Supplementary Figure $\mathbf{5}$. Analysis of miR845 family members and target TEs

816

817

818 during pollen development. a) Preferential sRNA size for miR845a and b during pollen development. b) Accumulation profile of miR845a- and miR845b-targeted TEs.

a.

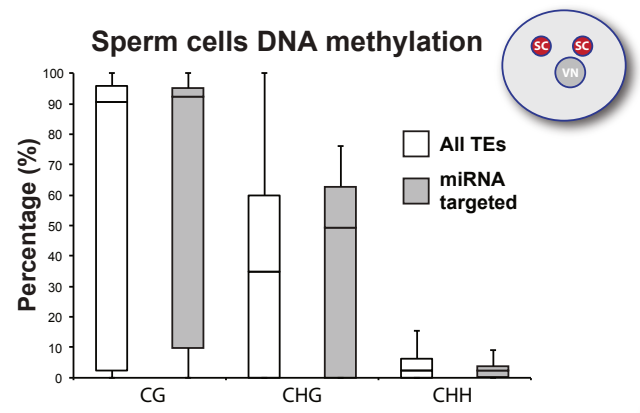

b.

Sperm cells DNA methylation
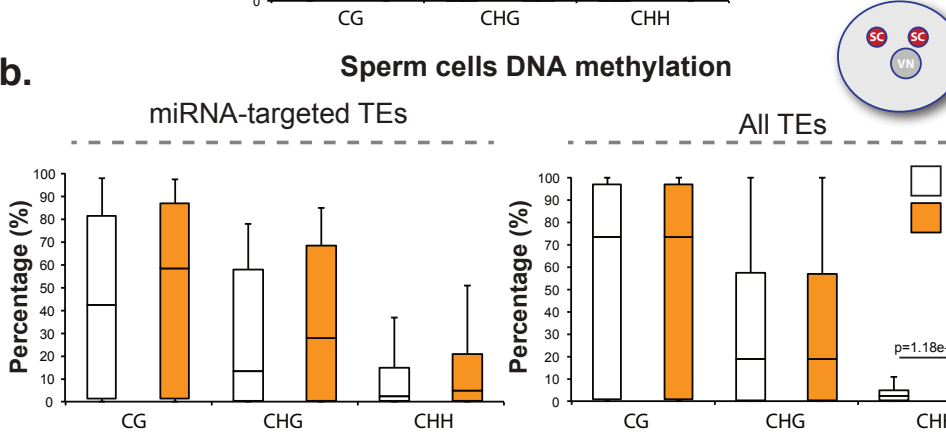

819

Supplementary Figure 6. a) Levels of cytosine methylation for the different

820 contexts (CG, CHG and $\mathrm{CHH}$ ) in the SCs for all TEs (white boxes) or miRNA-

822 targeted TEs (grey boxes). b) Levels of cytosine methylation for the different

823

824 contexts (CG, CHG and $\mathrm{CHH}$ ) in the SCs for miRNA-targeted TEs (left panel) or all TEs (right panel) in wild type (white boxes) and dme (orange boxes). Whiskers in the box plots extent to the maximum and minimum values. 
826

827

828

829

830

831

832

833

834

835

836 a.

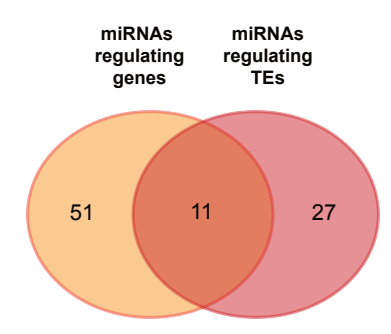

c.

$$
\begin{array}{r}
2500 \\
2000 \\
\sum_{0} 1500 \\
1000 \\
500 \\
0
\end{array}
$$$$
0
$$

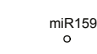

Common miRNAs

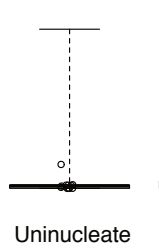

(Nu b.

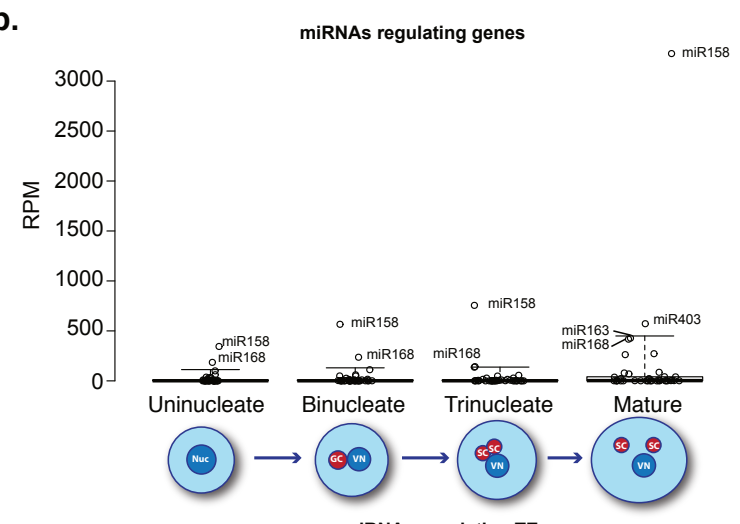

d.

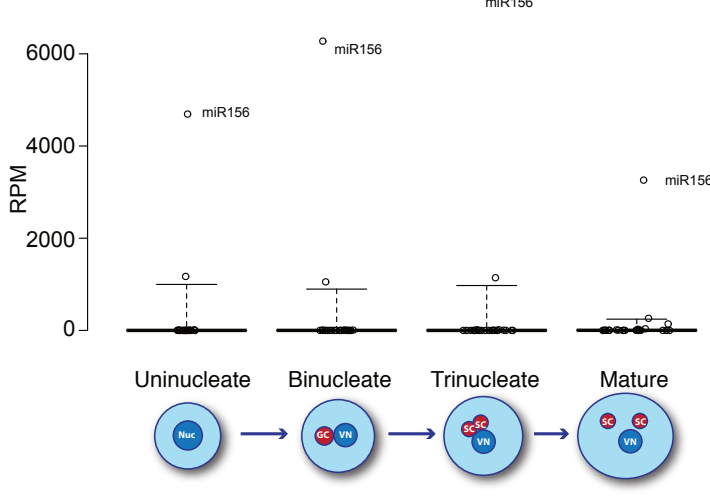

Supplementary Figure 7. a) Venn diagram depicting the overlap between the number of PARE-identifed miRNAs regulating genes and TEs. b-d) Box plots showing the accumulation values in reads per million (RPM) of PARE-identified miRNAs regulating genes (b), TEs (d) or both (c). Whiskers in the box plots extent to the $5^{\text {th }}$ and $95^{\text {th }}$ percentile.

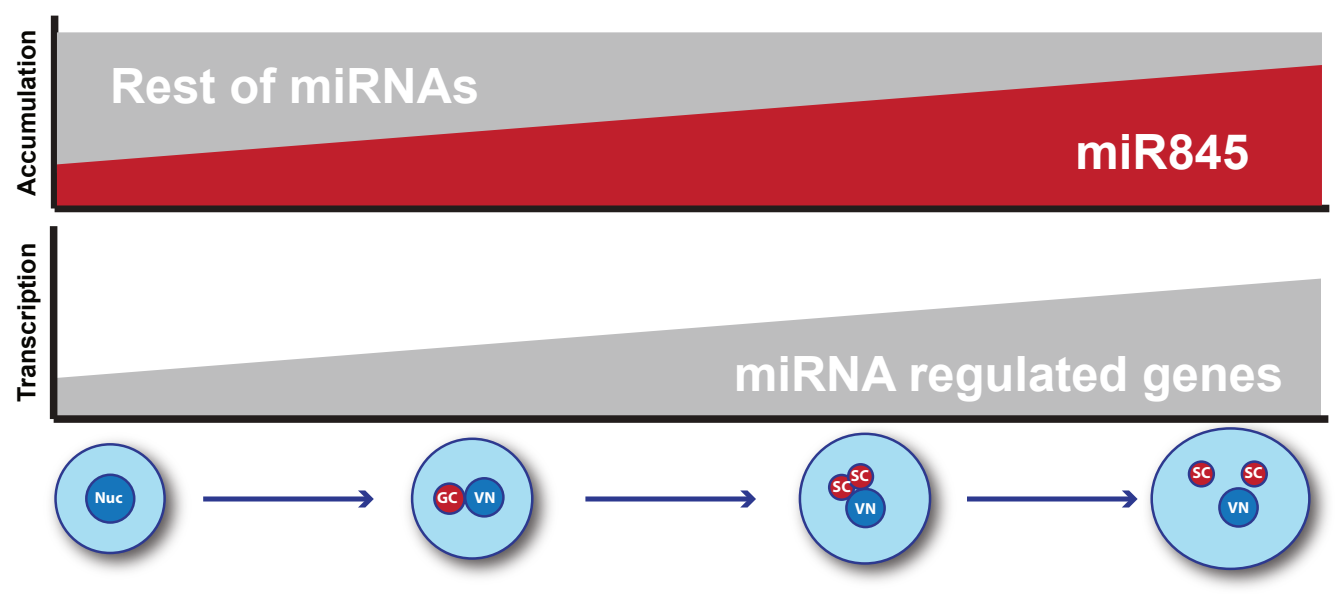

Supplementary Figure 8. Graphic conclusion. During pollen grain development the miRNome is reprogrammed to overload miR845 members at maturity.

Supplementary Table 1. Libraries produced in this study. 
838 Supplementary Table 2. Common and tissue specific miRNAs identified in this 839 study.

840

841 Supplementary Table 3. Pollen miRNA-targeted genes identified by PARE 842 sequencing.

843

844 Supplementary Table 4. Pollen miRNA-targeted TEs identified by PARE 845

846

847

848

849

850 sequencing.

Supplementary Table 5. Publicly available data analyzed in this study. Supplementary Table 6. Primers used in this study. 